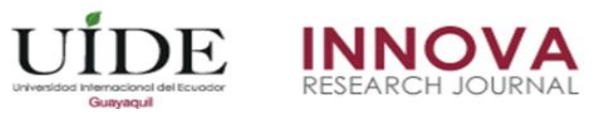

INNOVA Research Journal, ISSN 2477-9024

(Enero, 2018). Vol. 3, No.1 pp. 10-18

DOI: https://doi.org/10.33890/innova.v3.n1.2018.324

URL: http://revistas.uide.edu.ec/index.php/innova/index

Correo: innova@uide.edu.ec

\title{
Cansancio emocional, despersonalización y realización en trabajadores del sector bancario
}

\section{Emotional tiredness, depersonalization and realization in workers of the banking sector}

\author{
PhD. Antonio R. Gómez García \\ PhD. Pablo Suasnavas \\ Ing. Seg. Santiago F. Rodríguez V. \\ Universidad Internacional SEK Ecuador, Ecuador
}

Autor para correspondencia: antonio.gomez@uisek.edu.ec, pablo.suasnavas@uisek.edu.ec, santiago.rodriguez75@gmail.com

Fecha de recepción: 13 de Julio 2017 - Fecha de aceptación: 15 de Enero de 2018

Resumen: El estrés es un tema que cada día adquiere mayor importancia por los efectos dañinos que trae al hombre que lo padece y por el aumento de situaciones sociales, laborales, familiares y personales que lo provocan. En este trabajo se analizaron los componentes de cansancio emocional, despersonalización y realización personal con una muestra de 400 trabajadores del sector de la banca en Ecuador. Determinar los factores de influencia asociados al estrés laboral en Cajeros de una agencia bancaria del sector financiero. Se trata de un estudio descriptivo de corte transversal en los puestos de trabajo de Cajeros de una entidad bancaria ecuatoriana, durante el periodo comprendido entre el 27 de abril al 4 mayo de 2017; mediante el sistema de intranet corporativo. Los porcentajes de los Cajeros encuestas indican que el 10,00\% en cansancio emocional, el $12,00 \%$ en despersonalización y el $64,5 \%$ en realización personal, están categorizados en nivel elevado, pudiendo darse esto por la presión que día a día están sometidos, por las grandes cantidades de dinero que se manejan y el riesgo existente y por lo cotidiano de la actividad. Los resultados demostraron que un 27\% $(n=108)$ de los Cajeros a quienes se evaluó se encuentran con indicios de Burnout; es decir en un nivel alto de estrés.

Palabras clave: cansancio emocional; despersonalización y realización; estrés laboral; carga de trabajo; cajeros

Abstract: Stress is an issue that becomes increasingly important due to the harmful effects it brings to the man who suffers it and the increase in social, work, family and personal situations that cause it. In this work, the components of emotional exhaustion, depersonalization and personal fulfillment were analyzed with a sample of 400 workers from the banking sector in Ecuador. Determine the factors of influence associated with work stress in ATMs of a banking agency in the financial sector. This is a descriptive cross-sectional study of the ATMs of an Ecuadorian bank, during the period from April 27 to May 4, 2017; through the corporate intranet system. The percentages of cashier surveys indicate that $10.00 \%$ in emotional exhaustion, $12.00 \%$ in depersonalization and $64.5 \%$ in personal fulfillment, are categorized in a high level, this being possible due to the daily pressure they are subject, for the large amounts of money that are handled and the existing risk and for the daily activities. The results showed that $27 \%(\mathrm{n}=108)$ of the Cashiers to whom they were evaluated found evidence of Burnout; that is, at a high level of stress. Key Words: emotional tiredness; depersonalization and realization; work stress; workload 


\section{Introducción}

Burnout es un síndrome psicológico de ciertas experiencias interrelacionadas: primero es el agotamiento, como una respuesta al estrés. Pero además, en segundo lugar, es también un cambio, un cambio negativo sobre cómo se siente uno con el trabajo y las demás personas, cuando hablamos de ellas con indiferencial (cinismo), y tercero; cuando las personas se empiezan a sentir negativas sobre sí mismas, sobre su competencia, su capacidad, sus deseos y su motivación para trabajar. (García, 2014).

Seguramente, la gran mayoría de los seres humanos han sentido, alguna vez en su vida, que se han encontrado frente a una situación en su trabajo de mucha presión con la cual se les ha hecho imposible lidiar. Este es el famoso estrés laboral, el que se considera por muchos la epidemia o la enfermedad del siglo XXI. La sociedad actual ve cada vez con mayor naturalidad la existencia de este, y desgraciadamente algunos lo asumen como algo totalmente normal y como un factor propio de la vida cotidiana (Pérez \& Booth, 2009).

El ser humano diariamente está sometido a situaciones de presión que afecta especialmente los aspectos de la vida personal y laboral, sobre todo en los que realizan trabajos rutinarios, como es el caso del personal que labora como Cajeros en agencias bancarias debido a que cotidianamente se someten a tareas mentales y además al ambiente de presión de parte de los clientes.

Es frecuente que se perciba a los empleados bancarios como personas muy "estresadas”, por lo que nos preguntamos: ¿Pueden llegar esos niveles de estrés a hacerse crónicos, poniendo en riesgo la salud personal y el éxito organizacional?

Los altos estándares de calidad demandados actualmente en la prestación de servicios generan fuertes niveles de presión en sus prestatarios. En la prestación de servicios bancarios estas demandas van unidas a estrictos controles de seguridad, tanto por el manejo de valores como por las regulaciones de prevención de lavado de activos, y las regulaciones propias del sistema financiero (Peña \& Chico, 2007).

\section{Objetivo}

Debido a la presencia de estrés laboral en agencias bancarias, es el interés de este estudio poder determinar el grado de cansancio emocional, la despersonalización y la realización personal del personal de Cajeros de una entidad bancaria en Ecuador y exponer recomendaciones para enfrentar dicho fenómeno, de tal manera que los Cajeros puedan gozar de bienestar a nivel físico, psicológico y mejor rendimiento en la agencia bancaria donde laboran.

\section{Material - Métodos}

Se realizó un estudio descriptivo de corte transversal en los puestos de trabajo de ejecutivos transnacionales (cajeros) de una entidad bancaria ecuatoriana. 
Para la recolección de datos se aplicó el Maslach Burnout Inventory (MBI) (Maslach C, Jackson SE. Maslach Burnout Inventory. En: Seisdedos N, editor. Manual del Inventario Burnout de Maslach. Síndrome del "quemado" por estrés laboral asistencial. Madrid: TEA Ediciones; 1997. p. 5-28.) a 400 cajeros de 220 agencias a nivel nacional entre el 27 de abril al 4 mayo de 2017 mediante el sistema de intranet corporativo, respondieron 400 cajeros.

El estudio fue aprobado por la Gerencia General de la entidad bancaria a solicitud de la Jefatura de Seguridad y Salud Ocupacional. A los cajeros se les informó sobre los objetivos de estudio, el carácter voluntario y anonimato del mismo.

Para medir el grado de desgaste profesional se utilizó la versión española del MBI. El cuestionario se compone 22 preguntas dividido en tres dimensiones; Cansancio emocional (preguntas 1, 2, 3, 6, 8, 13, 14, 16 y 20), Despersonalización (preguntas 5, 10, 11, 15 y 22) y Realización personal (preguntas 4, 7, 9, 12, 17, 18, 19 y 21). La escala de respuestas para cada una de las preguntas es; nunca (0), pocas veces al año (1), una vez al mes (2), pocas veces al mes (3), una vez a la semana (4), pocas veces a la semana (5), todos los días (6).

Las puntuaciones finales para cada dimensión establecen el desgaste profesional en diferentes niveles; para el Cansancio emocional los valores se sitúan entre 0 y 54 ( $<15$ bajo, 1524 medio y >24 elevado), para la Despersonalización entre 0 y 30 ( $<4$ bajo, 4-9 medio y >9 elevado) y para la Realización personal entre 0 y 56 ( $<33$ bajo, 33-39 medio y >39 elevado) (Escribà V, Más R, Cárdenas M, Pérez S. Validación de la escala de estresores laborales en personal de enfermería: «the nursing stress scale». Gac Sanit. 1999; 13(3):191-200.).

Adicionalmente, se incluyeron variables socio-laborales; sexo, edad, antigüedad laboral como cajero en la entidad bancaria y horas trabajadas semanalmente.

Todos los cuestionarios se registraron en una base de datos Microsoft Excel y fueron analizados mediante Statistical Package for the Social Sciences (versión 24). Para el análisis estadístico se emplearon las frecuencia absolutas y relativas, media aritmética y desviación estándar, así mismo, se estableció la relación entre las variables de estudio y los niveles finales de cada dimensión mediante la Chi cuadrado de Pearson $(\mathrm{p}<0,05)$.

\section{Resultados}

En la Tabla 1 se muestra la distribución de los cajeros encuestados $(n=400)$ según sexo, edad, antigüedad en el puesto de trabajo y horas trabajadas semanalmente. Se observa un predominio de mujeres respecto a hombres, con una edad media de $29 \pm 7,1$ años.

Respecto a la antigüedad laboral como cajero en la entidad bancaria, el 78,6\% $(n=314)$ dispone de una permanencia de más de 1 año. El 89,5\% (n=358) de los cajeros trabaja más de 40 horas semanales.

Tabla 1. Distribución socio-laboral de los cajeros encuestados $(n=400)$.

\begin{tabular}{ccccccc}
\hline \multicolumn{2}{c}{ Hombre } & \multicolumn{2}{c}{ Mujer } & \multicolumn{2}{c}{ Total } \\
$\mathrm{N}$ & $\% \mathrm{n}$ & $\mathrm{n}$ & $\% \mathrm{n}$ & $\mathrm{n}$ & $\% \mathrm{n}$ \\
\hline
\end{tabular}




\begin{tabular}{|c|c|c|c|c|c|c|}
\hline \multicolumn{7}{|l|}{ Edad } \\
\hline $18-24$ & 34 & $27,2 \%$ & 70 & $25,5 \%$ & 104 & $26,0 \%$ \\
\hline $25-31$ & 58 & $46,4 \%$ & 133 & $48,4 \%$ & 191 & $47,8 \%$ \\
\hline $32-38$ & 17 & $13,6 \%$ & 47 & $17,1 \%$ & 64 & $16,0 \%$ \\
\hline $39-45$ & 5 & $4,0 \%$ & 18 & $6,5 \%$ & 23 & $5,8 \%$ \\
\hline $46-52$ & 9 & $7,2 \%$ & 5 & $1,8 \%$ & 14 & $3,5 \%$ \\
\hline 53-59 & 2 & $1,6 \%$ & 2 & $0,7 \%$ & 4 & $1,0 \%$ \\
\hline \multicolumn{7}{|c|}{ Antigüedad Laboral } \\
\hline$<1$ año & 35 & $28,0 \%$ & 51 & $18,5 \%$ & 86 & $21,5 \%$ \\
\hline 1 a 2 años & 21 & $16,8 \%$ & 52 & $18,9 \%$ & 73 & $18,3 \%$ \\
\hline 3 a 4 años & 30 & $24,0 \%$ & 74 & $26,9 \%$ & 104 & $26,0 \%$ \\
\hline$>5$ años & 39 & $31,2 \%$ & 98 & $35,6 \%$ & 137 & $34,3 \%$ \\
\hline \multicolumn{7}{|l|}{ Horas Semanales } \\
\hline$<40$ & 1 & $0,8 \%$ & 3 & $1,1 \%$ & 4 & $1,0 \%$ \\
\hline 40 & 7 & $5,6 \%$ & 31 & $11,3 \%$ & 38 & $9,5 \%$ \\
\hline$>40$ & 117 & $93,6 \%$ & 241 & $87,6 \%$ & 358 & $89,5 \%$ \\
\hline
\end{tabular}

En la Tabla 2 se muestra los resultados de los sentimientos, actitudes y desgaste profesional del personal de Cajeros durante su jornada de trabajo, dando como resultado en el factor de Cansancio emocional, que valora la vivencia de estar exhausto emocionalmente por las demandas de trabajo, un $10 \%$ entre hombres y mujeres $\tilde{n}(n=40)$ se encuentran en un nivel Elevado, un 20,4\% $(n=56)$ en mujeres y 21,6\% $(n=27)$ en hombres están en un nivel Medio; mientras que un $71,6 \%(n=197)$ en mujeres y $64 \%(n=80)$ en hombres se encuentran en un nivel Bajo.

Los resultados del factor Despersonalización que valora el grado en que cada uno reconoce actitudes de frialdad y distanciamiento, pone en evidencia que un $12 \%$ entre mujeres y hombres $(n=48)$ se encuentran en un nivel Elevado, un 34\% $(n=94)$ en mujeres y 35,3\% $(n=44)$ en hombres están en un nivel Medio; mientras que un 56,7\% ( $\mathrm{n}=156)$ en mujeres y 46,4\% ( $\mathrm{n}=58)$ en hombres se encuentran en un nivel Bajo.

Finalmente los resultados del factor Realización personal que evalúa los sentimientos de autoeficiencia y realización personal en el trabajo indican un 64,5\% ( $\mathrm{n}=258)$ entre hombres y mujeres se encuentran en un nivel Elevado, un 29,8\% ( $n=82)$ en mujeres y 30,4\% ( $n=38)$ en hombres se encuentran en un nivel Medio; mientras que un 5,8\% $(n=16)$ en mujeres y 4,8\% $(n=6)$ en hombres se encuentran en un nivel bajo.

Tabla 2. Frecuencia e intensidad de los factores del Síndrome de Burnout.

\begin{tabular}{|c|c|c|c|c|c|c|c|c|}
\hline & \multicolumn{2}{|c|}{ Hombre } & \multicolumn{2}{|c|}{ Mujer } & \multicolumn{2}{|c|}{ Total } & \multirow{2}{*}{$\begin{array}{l}\text { Medi } \\
\text { a }\end{array}$} & \multirow{2}{*}{$\begin{array}{l}\text { Desviació } \\
\text { n Estándar }\end{array}$} \\
\hline & $\mathrm{n}$ & $\% n$ & $\mathrm{~N}$ & $\% n$ & $\mathrm{n}$ & $\%$ n & & \\
\hline $\begin{array}{l}\text { Cansancio } \\
\text { Emocional }\end{array}$ & & & & & & & 11,8 & 9,5 \\
\hline Bajo & $0^{8}$ & $\begin{array}{l}64,0 \\
\%\end{array}$ & $\begin{array}{l}19 \\
7\end{array}$ & $\begin{array}{l}71,6 \\
\%\end{array}$ & $\begin{array}{l}27 \\
7\end{array}$ & $\begin{array}{l}69,3 \\
\%\end{array}$ & & \\
\hline
\end{tabular}




\begin{tabular}{|c|c|c|c|c|c|c|c|c|}
\hline Medio & $7^{2}$ & $\begin{array}{l}21,6 \\
\%\end{array}$ & 56 & $\begin{array}{l}20,4 \\
\%\end{array}$ & 83 & $\begin{array}{l}20,8 \\
\%\end{array}$ & & \\
\hline Elevado & $8^{1}$ & $\begin{array}{l}14,4 \\
\%\end{array}$ & 22 & $8,0 \%$ & 40 & $\begin{array}{l}10,0 \\
\%\end{array}$ & & \\
\hline $\begin{array}{l}\text { Despersonalizaci } \\
\text { ón }\end{array}$ & & & & & & & 3,7 & 4,6 \\
\hline Bajo & $8^{5}$ & $\begin{array}{l}46,4 \\
\%\end{array}$ & $\begin{array}{l}15 \\
6\end{array}$ & $\begin{array}{l}56,7 \\
\%\end{array}$ & $\begin{array}{l}21 \\
4\end{array}$ & $\begin{array}{l}53,5 \\
\%\end{array}$ & & \\
\hline Medio & $\begin{array}{l}4 \\
4\end{array}$ & $\begin{array}{l}35,2 \\
\%\end{array}$ & 94 & $\begin{array}{l}34,2 \\
\%\end{array}$ & $\begin{array}{l}13 \\
8\end{array}$ & $\begin{array}{l}34,5 \\
\%\end{array}$ & & \\
\hline Elevado & $3^{2}$ & $\begin{array}{l}18,4 \\
\%\end{array}$ & 25 & $9,1 \%$ & 48 & $\begin{array}{l}12,0 \\
\%\end{array}$ & & \\
\hline $\begin{array}{l}\text { Realización } \\
\text { Personal }\end{array}$ & & & & & & & 39,6 & 3,8 \\
\hline Bajo & 6 & $4,8 \%$ & 16 & $5,8 \%$ & 22 & $5,5 \%$ & & \\
\hline Medio & $8^{3}$ & $\begin{array}{l}30,4 \\
\%\end{array}$ & 82 & $\begin{array}{l}29,8 \\
\%\end{array}$ & $\begin{array}{l}12 \\
0\end{array}$ & $\begin{array}{l}30,0 \\
\%\end{array}$ & & \\
\hline Elevado & $\begin{array}{l}8 \\
1\end{array}$ & $\begin{array}{l}64,8 \\
\%\end{array}$ & $\begin{array}{l}17 \\
7\end{array}$ & $\begin{array}{l}64,4 \\
\%\end{array}$ & $\begin{array}{l}25 \\
8\end{array}$ & $\begin{array}{l}64,5 \\
\%\end{array}$ & & \\
\hline
\end{tabular}

\begin{tabular}{|l|l|l|l|l|}
\hline Escala & \multicolumn{1}{|c|}{ Item } & Baja & Media & Alta \\
\hline CE & $1,2,3,6,8,13,14,16,20$ & $<18$ & $19-27$ & $>27$ \\
\hline DP & $5,10,11,15,22$ & $<6$ & $6-9$ & $>9$ \\
\hline RP & $4,7,9,12,17,18,19,21$ & $<33$ & $34-39$ & $>40$ \\
\hline
\end{tabular}

Figura 1. Escala Maslach Burnout Inventory

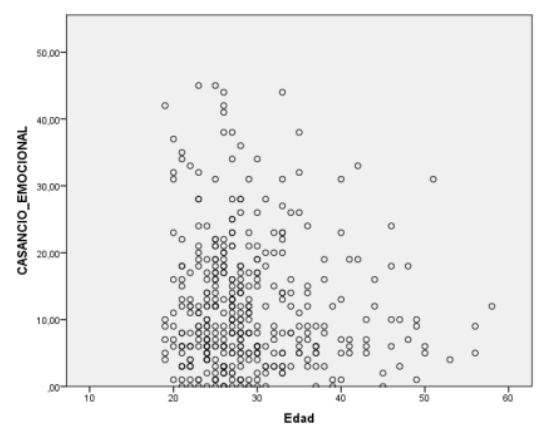

Figura 2. Cansancio Emocional

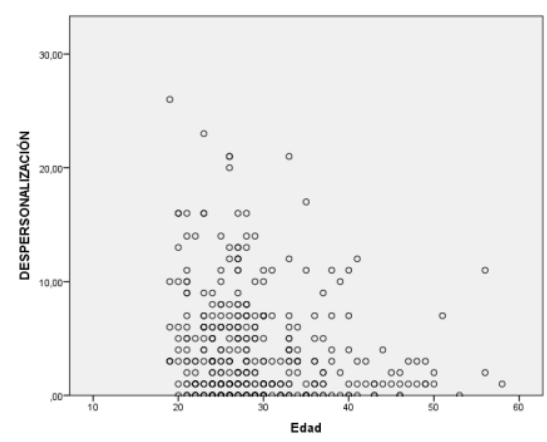

Figura 3. Despersonalización. 


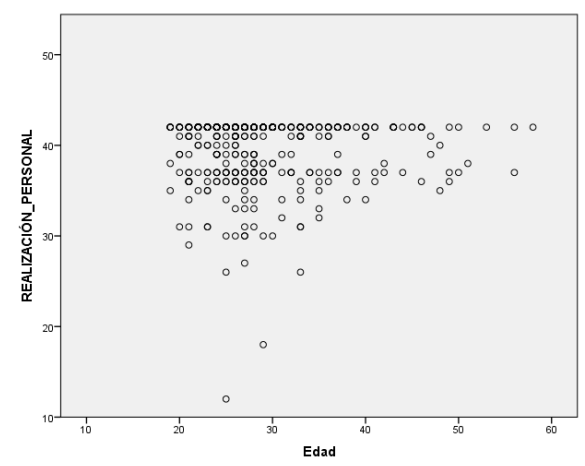

Figura 4. Realización personal.

\section{Discusión}

Este análisis pretende ayudar a los empleados de la agencia bancaria analizada para que pueda manejar y enfrentar el estrés y de esa manera brinden un mejor servicio a los usuarios, se observó que muchos de ellos presentan un nivel de estrés medio, sabiendo en que es natural debido a las tareas que a diario desempeñan.

Los resultados nos reflejaron que existe 34 Cajeros que tienen indicios de Burnout en cansancio emocional, 48 Cajeros presentan indicios de Burnout en despersonalización y 26 Cajeros presentan indicios de Burnout en realización personal, pudiendo darse esto por la presión que día a día están sometidos, por la atención a clientes, por las grandes cantidades de dinero que se manejan, el riesgo existente y por lo cotidiano de la actividad.

Es normal que los empleados de las instituciones bancarias se estresen debido a la carga de trabajo, esto provoca que las personas o empleados comiencen a presentar síntomas de estrés como cansancio, tensión, irritabilidad entre otros síntomas que se van agravando y que van afectando el desenvolvimiento laboral. Asimismo, a largo plazo el estrés puede provocar deterioro cognitivo. Las prisas, la falta de tiempo o la tensión aumentan las conductas no saludables.

Los efectos del estrés en el trabajo son las enfermedades crónicas más difíciles de diagnosticar, ya que estas enfermedades requieren un largo período de desarrollo que se pueden ver influidas en la manera de cómo los cajeros atienden a los usuarios que visitan las instituciones bancarias donde se realizó la investigación.

\section{Conclusiones}

Los resultados demostraron que un 27\% $(n=108)$ de los Cajeros a quienes se evaluó se encuentran con indicios de Burnout; es decir en un nivel alto de estrés.

Falta trabajar en la autoestima de los cajeros, puesto que el nivel de falta de realización personal es muy elevado, lo que impide el desempeño adecuado en su trabajo. 
Las autoridades de las agencias bancarias donde se realizó la investigación deben preocuparse no solo por la salud de los empleados sino también por el beneficio de la institución.

Las empresas que ayudan a los trabajadores a afrontar el estrés y a mantener un ambiente adecuado en el trabajo tienen más posibilidades de lograr metas. Los gastos y pérdidas derivadas por el costo del estrés aumenta año con año, determinados por los índices de ausentismo, baja productividad, accidentes profesionales, baja motivación y lo que es más importante la incidencia sobre la salud física y psicológica de las personas.

Existen factores que inciden negativamente sobre las actividades, el rendimiento y la satisfacción laboral, como: El temor a la autoridad que en general lo hace trabajar en un estado de tensión, y que en ciertos casos limita la eficiencia, en ocasiones se necesitan períodos prolongados de adaptación por características individuales de los trabajadores, la falta de atención al personal hace que el trabajador sienta que es privado de individualidad.

\section{Bibliografía}

"García, A. (2014). Entrevista con Christina Maslach: reflexiones sobre el síndrome de Burnout. Revista Liberabit, vol. 20 no. 2.

"Pérez \& Booth (2009). El estrés ocupacional en personas que desempeñan 28 medios y altos en instituciones del sector financiero de la ciudad de Valdivia. Tesina de grado.

"Peña \& Chico (2007). Prevalencia del síndrome de "quemarse por el trabajo" burnout, en empleados de sucursales de un banco dominicano Ciencia y Sociedad. Revista Ciencia y Sociedad. 645-667.

"Maslach, C. (1997). Síndrome del "quemado" por estrés laboral asistencial. Manual del Inventario Burnout de Maslach. P. 5-28.

“Escribá, V., Más, R., Cárdenas, M., \& Pérez, S. (1999). Validación de la escala de estresores laborales en personal de enfermería. Gac Sanit. 191-200.

\section{Anexo}

Instrucciones: Marca con una X la intensidad que corresponda a tu situación.

Señale la respuesta que crea oportuna sobre la frecuencia con que siente los enunciados:

$0=$ nunca. $1=$ pocas veces al año o menos. $2=$ una vez al mes o menos.

$3=$ unas pocas veces al mes. $4=$ una vez a la semana. $5=$ pocas veces a la semana. $6=$ todos $\operatorname{los}$ días.

$1 \quad$ Me siento emocionalmente agotado por mi trabajo

(0) (1) (2) (3) (4) (5) (6)

2 Cuando termino mi jornada de trabajo me siento vacío.

(0) (1) (2) (3) (4) (5) (6)

3 Cuando me levanto por la mañana y me enfrento a otra jornada de trabajo me siento fatigado. 
(0) (1) (2) (3) (4) (5) (6)

4 Siento que puedo entender fácilmente a los clientes.

(0) (1) (2) (3) (4) (5) (6)

5 Siento que estoy tratando a algunos clientes como si fueran objetos impersonales.

(0) (1) (2) (3) (4) (5) (6)

6 Siento que trabajar todo el día con la gente me cansa.

(0) (1) (2) (3) (4) (5)

$7 \quad$ Siento que trato con mucha eficacia los problemas de mis clientes.

(0) (1) (2) (3) (4) (5) (6)

8 Siento que mi trabajo me está desgastando.

(0) (1) (2) (3) (4) (6)

9 Siento que estoy influyendo positivamente en la vida de otras personas a través de mi trabajo.

(0) (1) (2) (3) (4) (5) (6)

10 Siento que me he hecho más duro con la gente

(0) (1) (2) (3) (4) (5) (6)

11 Me preocupa que este trabajo me esté endureciendo emocionalmente.

(0) (1) (2) (3) (4) (5) (6)

12 Me siento con mucha energía en mi trabajo.

(0) (1) (2) (3) (4) (5) (6)

13 Me siento frustrado en mi trabajo.

(0) (1) (2) (3) (4) (5) (6)

14 Siento que estoy demasiado tiempo en mi trabajo.

(0) (1) (2) (3) (4) (5) (6)

15 Siento que realmente no me importa lo que les ocurra a mis clientes.

(0) (1) (2) (3) (4) (5) (6)

16 Siento que trabajar en contacto directo con la gente me cansa.

(0) (1) (2) (3) (4) (5) (6)

17 Siento que puedo crear con facilidad un clima agradable con mis clientes.

(0) (1) (2) (3) (4) (5) (6)

18 Me siento estimado después de haber trabajado íntimamente con mis clientes.

(0) (1) (2) (3) (4) (5) (6)

19 Creo que consigo muchas cosas valiosas en este trabajo.

(0) (1) (2) (3) (4) (5) (6)

20 Me siento como si estuviera al límite de mis posibilidades.

(0) (1) (2) (3) (4) (5) (6)

21 Siento que en mi trabajo los problemas emocionales son tratados de forma adecuada.

(0) (1) (2) (3) (4) (5) (6) 
22 Me parece que los clientes me culpan de alguno de sus problemas.

(0) (1) (2) (3) (4) (5) (6) 